\title{
Chagas disease 101
}

\section{It is 101 years since Carlos Chagas discovered the parasite responsible for the disease that now bears his name. What progress has been made since this discovery? Here Julie Clayton gives the low-down on Chagas disease.}

\section{What is Chagas disease?}

Chagas disease is a condition caused by the parasite Trypanosoma cruzi, which enters the body through broken skin and mucous membranes, and causes acute but often mild symptoms such as fever, swelling of lymph nodes and tissues, conjunctivitis and skin lesions. In poor rural areas of South and Central America, where the disease is endemic and health-care facilities are inadequate, these symptoms go largely unattended. Without treatment, the acute infection spontaneously subsides in the majority of patients; however, the chronic infection can persist unnoticed for $>30$ years before causing complications such as abnormal heart rhythm, heart failure, digestive problems and sudden cardiac death. About one in three carriers develop these chronic symptoms, which can be the first time they are diagnosed with T. cruzi infection.

The Brazilian physician Carlos Chagas identified T. cruzi as the causative agent of the disease in 1909, and later determined its clinical manifestations, epidemiology and entire life cycle in vectors, wild reservoirs and human hosts. Controversially, Chagas was never awarded a Nobel prize, despite being nominated twice.

\section{Where is Chagas disease found?}

T. cruzi is endemic to Latin America, affecting 10-12 million people and killing $>15,000$ each year ${ }^{1,2}$. It is also carried by hundreds of thousands of people in Europe (mostly Spain and Portugal), the United States, Canada, Japan and Australia. The carriers are typically South American immigrants, who are often unaware of their infection, which has significant public-health implications for the management of blood banks and health-care provision, for example (see Chagas disease: a new worldwide challenge on page S6 and Who, how, what and where? on page S8).

\section{How is Chagas disease transmitted?}

The parasite T. cruzi moves among mammals, humans and triatomine or 'kissing bug' insects during its life cycle (Fig. 1). There are $>100$ species of triatomine bugs with the potential to transmit T. cruzi, three of which are significant domestic species, including Triatoma infestans. Animal hosts in endemic areas include bats, palm tree-dwelling opossums, dogs, rats, guinea pigs and other small mammals, and, in the United States, opossums and raccoons are naturally infected with T. cruzi. Triatomine bugs live in palm trees, hollow trees, burrows, rock piles or other refuges, and feed on the blood of diverse mammals and other vertebrates, and on humans with whom they come into contact by flying into and colonizing their dwellings. Houses that are made of mud-brick - which are common in some poor areas contain many cracks and crevices in which the bugs can hide.

A triatomine picks up the parasitic infection by feeding on the blood of an infected animal or human. Once inside, the parasite divides rapidly in the insect gut before emerging in its faeces. When the triatomine takes another blood meal, it defecates on the skin of the victim, depositing T. cruzi in the process. The parasite is able to penetrate the new host if the skin is broken - by the bite of the bug or through other cuts and abrasions - or through the soft skin of the eyes and mouth. It can

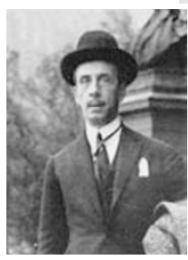
infect the cells of the soft tissues below, and then enter the blood stream and circulate to reach other body tissues. The parasite is not fussy - it can infect all mammalian cells.

Alternatively, people and animals can become infected orally through ingesting food and drink that is contaminated with the faeces of a triatomine carrying the parasite. This happened recently at a school in Caracas, Venezuela, where there were 75 cases of acute Chagas disease and one death among 103 infected children following the consumption of contaminated Guava juice ${ }^{3}$. The fruit of the açaí palm is another common source of contamination, as it is harvested and pressed in the Amazon forest.

Triatomine bugs do not exist in Europe and are less common in North America, but people can still pass on the infection to others through blood or organ donation. Mothers can also unwittingly pass the parasite onto their babies at birth, which is likely to affect infants worldwide (see Chagas disease: a new worldwide challenge on page S6).

\section{What is so special about T. cruzi?}

T. cruzi poses an extra challenge for those trying to develop new drugs for Chagas disease because it is an intracellular parasite: it hijacks the cellular machinery to produce hundreds of new parasites until the cell bursts, releasing the progeny to infect surrounding tissue. This means that drugs against the parasite need to be able to cross the plasma membrane in order to kill it. By contrast, the other trypanosome parasites Leishmania (which causes leishmaniasis) and Trypanosoma brucei (which causes African trypanosomiasis or sleeping sickness) remain in the blood, and so are potentially more accessible to drugs. All of these are neglected tropical diseases for which there has been little investment in drug research and development (R\&D).

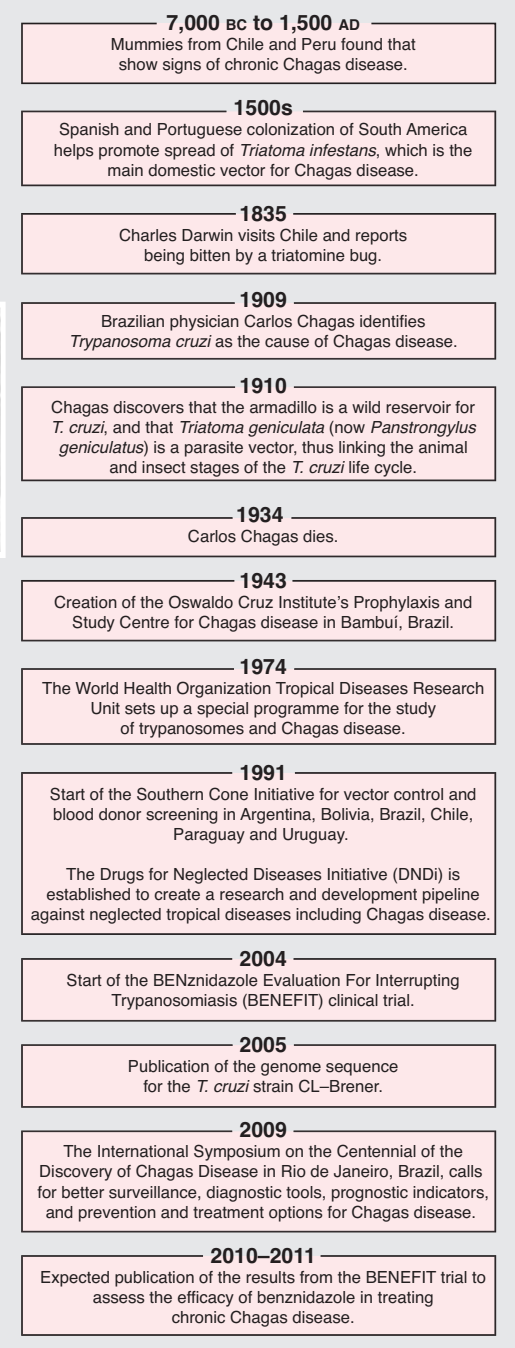




\section{Trypanosomiasis, American (Chagas disease) \\ (Trypanosoma cruzi)}

Triatomine Bug Stages

Triatomine bug takes a blood meal

1 (passes metacyclic trypomastigotes in feces,

trypomastigotes enter bite wound or

What treatments are available?

The only existing options for $>40$ years have been the drugs benznidazole and nifurtimox. These are especially effective at curing infection in the early, acute phase, provided that patients complete a full 60-day course at the correct dose, with cure rates of up to $80 \%$. The drugs are considered less effective in patients who have been chronically infected for $>10$ years - although this is the subject of considerable debate. Benznidazole and nifurtimox are not ideal, given that their side effects can be severe, and they can lead to resistance. Consequently, many physicians prefer not to prescribe these drugs, and many harbour the belief that they might be useless anyway.

\section{What is the controversy around treating chronic Chagas disease?}

In people who are chronically infected, the parasite often seems to disappear. For decades, the traditional diagnostic methods were examination of blood samples by microscopy, and (for research purposes) laboratory culture of blood samples and xenodiagnosis, in which blood is fed to triatomine bugs whose faeces are then analysed for the presence of T. cruzi. These methods frequently failed to detect T. cruzi in the blood of patients with chronic heart problems or other complications. Yet serum antibodies could still be detected. These observations led people to conclude that chronic Chagas disease is an autoimmune condition, which is sparked initially by the parasite but then becomes self-sustaining, as the immune system goes on to attack the body tissues.

The use of more sensitive immunohistochemical and PCR-based diagnostic tests has now overturned the autoimmune hypothesis showing that T. cruzi hides deep in the heart and other tissues (as found at post-mortem ${ }^{4,5}$ ), and can circulate in the blood in relatively low numbers, which coincides with chronic complications. Yet clinicians still lack clear guidelines on whether and when to treat chronic carriers, particularly those aged $>50$ years.

\section{Why is Chagas disease so neglected?}

As well as the controversies over its pathology, Chagas disease has suffered from an inadequate $R \& D$ pipeline. Many commentators attribute this to a lack of interest from pharmaceutical firms in producing drugs for diseases that mostly affect the world's poorest. There also appears to be a lack of government commitment - even in many countries where Chagas disease is endemic - and therefore little investment in basic research and infrastructure for clinical trials. The Global Funding of Innovation for Neglected Diseases (G-FINDER) survey of 2009 highlights that, of the US\$132 million spent globally in 2008 on diseases caused by kinetoplastid parasites (including T. cruzi), only US\$15.6 million was invested in Chagas disease R\&D. Most of that was for basic research, with less than US $\$ 5$ million being used for new diagnostics, drugs and vaccines.

\section{A new chance for benznidazole?}

Late 2010 or early 2011 will see the results of the largest clinical trial yet for Chagas disease:

\section{Did Darwin have Chagas disease?}

Charles Darwin wrote in his journal The Voyage of the Beagle about having been bitten by the Triatoma kissing bug when visiting Chile in 1835, and subsequently suffering poor health, including vomiting gut pain, headaches and depression, for the rest of his life. The presence of gastric symptoms and his ultimate demise from cardiac problems have led some scholars to propose that Darwin was suffering from Chagas disease, although certain other factors suggest otherwise - including the fact that the parasite is not transmitted directly in the bite of the insect. The many other explanations put forward include stress, allergies and lactose intolerance - an inability to digest milk and dairy products, made worse by Darwin's love of cream. The truth might be unattainable without exhuming Darwin's body from Westminster Abbey in London, United Kingdom, to look for the presence of Trypanosoma cruzi DNA.

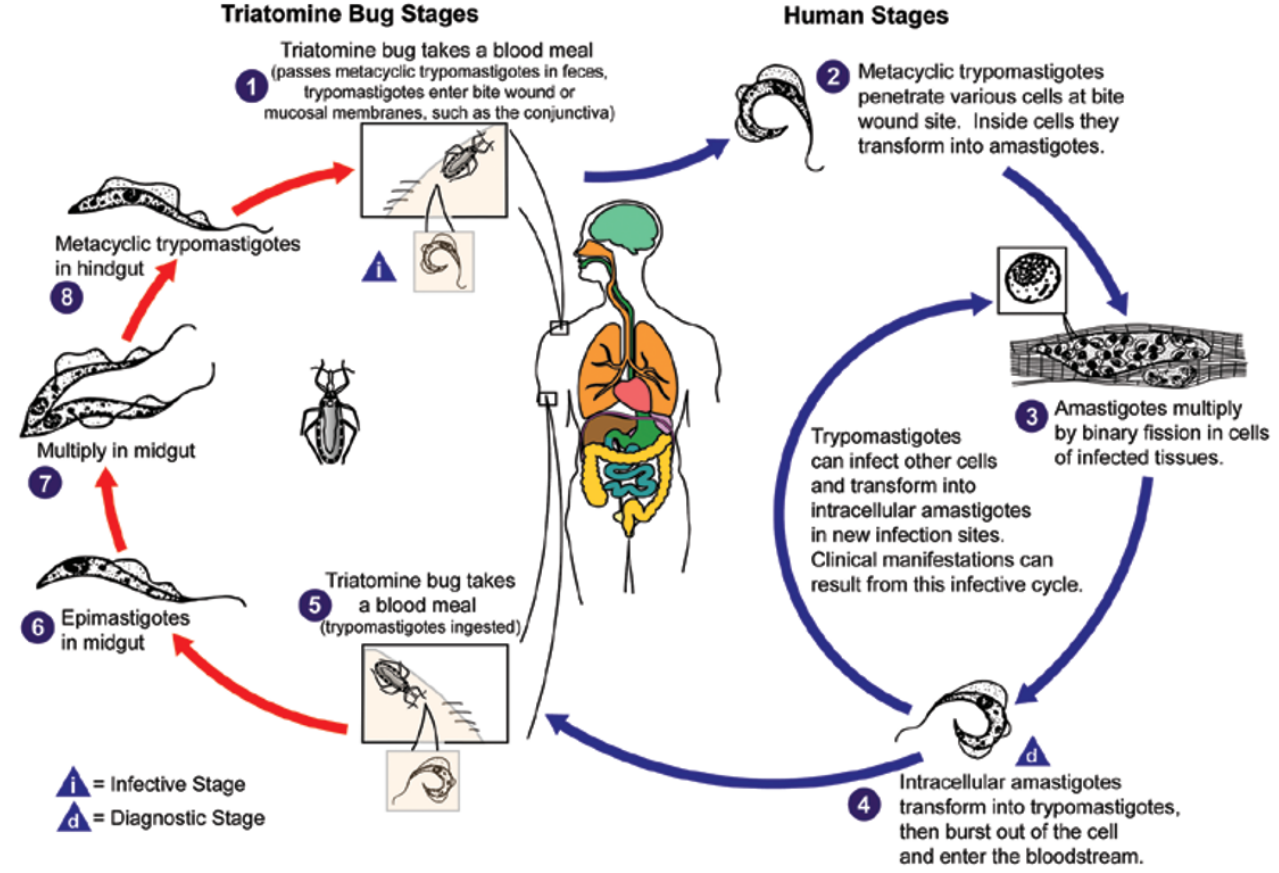

Fig. $1 \mid$ Life cycle of Trypanosoma cruzi, the causal agent of American trypanosomiasis. Image courtesy of Centers for Disease Control and Prevention, A. J. da Silva and M. Moser.

the BENznidazole Evaluation For Interrupting Trypanosomiasis (BENEFIT) trial. The trial started in 2004 in Argentina, Brazil and Colombia with the goal of recruiting 3,000 patients with chronic Chagas disease, and aims to provide clearer guidance on the benefits of giving benznidazole to long-term chronic patients.

BENEFIT uses real-time PCR and serological tests, and aims to provide precise figures on the cure rate with benznidazole in chronic disease. More importantly, it will test whether curing Chagas disease - or even just reducing the parasite load - can prevent or reduce the severity of cardiac complications, which are the major cause of death.

However, technical obstacles remain that have hampered drug research in the past, particularly the difficulty of demonstrating treatment success (cure or no cure). It is easier to show treatment failure - that is, the reactivation of T. cruzi and the appearance of drugresistant parasite. Overcoming these obstacles will be an important goal in the coming years if significant progress is to be made against Chagas disease.

Julie Clayton is a freelance writer based in Bristol, United Kingdom.

1. World Expert Committee. World Heath Organ. Tech. Rep. Ser. 905, i-vi, 1-109 (2002).

2. Jannin, J. \& Salvatella, R. Quantitative Estimates of Chagas Disease in the Americas [OPS/HDM/CD/425-06]1-28 (Pan American Health Organization, 2006).

3. Alarcón de Noya, B. et al. J. Infect. Dis. 201, 1308-1315 (2010).

4. Jones, E. M. et al. Am. J. Trop. Med. Hyg. 48, 348-357 (1993).

5. Schijman, A. G. et al. Am. J. Trop. Med. Hyg. 70, 210-220 (2004). 